\title{
Optics
}

\section{Optics and spectroscopy of exciton states in quasi - zero - dimensional nanostructures: Theory}

which consists of two reviews:

\author{
Exciton states spectroscopy in quasi - zero - dimensional nanostructures: Theory
}

and

\author{
Theory of excitons and excitonic quasimolecules formed from spatially separated \\ electrons and holes in quasi - zero - dimensional nanosystems
}

\author{
Sergey I. Pokutnyi \\ Chuiko Institute of Surface Chemistry, National Academy of Sciences of Ukraine, 17 General Naumov Str., UA - 03164 Kyiv, Ukraine
}

\section{Email address:}

Pokutnyi_Sergey@inbox.ru

\section{To cite this article:}

Sergey I. Pokutnyi. Optics and Spectroscopy of Exciton States in Quasi - Zero - Dimensional Nanostructures: Theory. Optics. Special Issue: Optics and Spectroscopy of the Charge Carriers and Excitons States in Quasi - Zero - Dimensional Nanostructures.

Vol. 3, No. 6-1, 2014, pp. 1-1. doi: 10.11648/j.optics.s.2014030601.11

\begin{abstract}
The review analyzes the results of theoretical investigations of excitons states (electron - hole pairs states) in a quasi - zero - dimensional nanosystems consisting of spherical semiconductor nanocrystals (quantum dots) placed in transparent dielectric matrices. The theory of exciton states in a quantum dot under conditions of dominating polarization interaction of an electron and a hole with a spherical (quantum dot - dielectric matrix) interface are developed. An shown, that the energy spectrum of heavy hole in the valence band quantum dot is equivalent to the spectrum of hole carrying out oscillator vibrations in the adiabatic electron potential. We show that the absorption and emission edge of quantum dots is formed by two transitions of comparable intensity from different hole size - quantization levels and into a lower electron size - quantization level. Propose a theoretical prospect of using hole transitions between equidistant series of quantum levels observed in nanocrystals for desining a nanolaser. The theory of an exciton formed from spatially separated electron and hole (the hole is in the quantum dot volume, and the electron is localized at the outer spherical quantum dot-dielectric matrix interface) is developed within the modified effective mass method. The effect of significantly increasing the exciton binding energy in quantum dots of zinc selenide, synthesized in a borosilicate glass matrix, relative to that in a zinc selenide single crystal is revealed. We developed the theory of a biexcitons formed from spatially separated electrons and holes (the hole is in quantum dot volume, and the electron is localized at the outer surface of the quantum dot - dielectric matrix interface) in a nanosystem that consists of zinc selenide quantum dots synthesized in a glassy matrix.
\end{abstract}

Keywords: Spectroscopy, Exciton States, Energy Spectrum, Exciton Binding Energy, Absorption of Light, Optical Nanolaser, Biexcitons, Semiconductor Quantum Dots 